Arthroskopie 2021 · 34:94-102

https://doi.org/10.1007/s00142-020-00429-4

Angenommen: 11. Dezember 2020

Online publiziert: 8. Januar 2021

(c) Springer Medizin Verlag GmbH, ein Teil von Springer Nature 2021

\section{Redaktion}

W. Petersen, Berlin

A. Voss, Regensburg

Wolf Petersen - Tillmann Hees - Julia Ohde

Sportklinik Berlin und Klinik für Orthopädie und Unfallchirurgie, am Martin Luther Krankenhaus, BerlinGrunewald, Berlin, Deutschland

\title{
Aktueller Stand virtueller Simulatoren für die chirurgische Ausbildung in Orthopädie und Unfallchirurgie
}

30]. Erschwerte Verhältnisse, wie z. B. ein enges Gelenk oder schlechte Sicht, können das Fehlerrisiko außerdem erhöhen.

\section{》) Die Lernkurve bei \\ arthroskopischen Operationen ist flach}

Aufgrund dieser komplexen Anforderungen ist die Lernkurve bei arthroskopischen Operationen flach [3, 4, 11, 16, 17, 27]. Daher ist das Erlernen arthroskopischer Operationen mit vergleichsweise längeren Operationszeiten [15] und höheren Komplikationsraten verbunden [12] als das Erlernen offener Operationen.

Das traditionelle chirurgische Training (Ausbildung der Assistenzärzte im Operationssaal) kann bei arthroskopischen Operationen aus o.g. Gründen sehr zeitintensiv sein, da bestimmte taktile Fertigkeiten nicht theoretisch vermittelt werden können. Diese Fertigkeiten („skills“) müssen trainiert werden.

Daher werden in der arthroskopischen Ausbildung schon seit längerer Zeit Simulatoren eingesetzt. Dabei handelt es sich um Kniemodelle aus Kunststoff, in denen mit Kamera und Instrumenten arthroskopische Operationen simuliert werden können (Arbeiten mit Instrumenten unter Triangulation). Die anatomische Genauigkeit dieser Modelle und die haptischen Eigenschaften von Gelenkkapsel, Knorpel und Menisken entsprechen denen des normalen Kniegelenks jedoch nicht. Auch der Erfolg der arthroskopischen Übungen ist an diesen Modellen nur über das Messen der Operationszeit zu evaluieren. Es fehlen andere Möglichkeiten zur Rückkopplung zu Erfolgskriterien wie iatrogenen Knorpelschäden, Wegstrecke der Instrumente etc.

Eine weitgehend normale Anatomie bieten Kniegelenke von Körperspendern. Aber auch hier bestehen Nachteile wie eine limitierte Verfügbarkeit, hohe Kosten, ein fehlender Muskeltonus oder die hohe Prävalenz degenerativer Veränderungen am Präparat. Auch hier ist die Erfassung von Erfolgskriterien auf die Zeit oder subjektive Scores limitiert.

Diese Lücke wurde in den letzten Jahren durch die Entwicklung virtueller Simulatoren geschlossen [27]. Diese Modelle etablieren sich zunehmend in der arthroskopischen Ausbildung auf Arthroskopiekursen oder auch in der klinischen Ausbildung im Krankenhaus (- Abb. 1). Insbesondere bei jungen Kollegen, die erfahrungsgemäß durch das Gaming mehr Erfahrung mit der virtuellen Realität (VR) haben, stoßen diese neuen Möglichkeiten auf hohes Interesse.

In Anbetracht der gegenwärtigen Kosten für diese Modelle stellt sich trotz aller Begeisterung für technische Neuerungen natürlich schnell die Frage nach dem wissenschaftlichen Nutzen. So wird die Effizienz virtueller Arthroskopiesimulatoren auch von einigen Autoren in Frage gestellt [2]. In anderen Berufszweigen mit hohem Risikopotenzial gehört das Simulationstraining jedoch schon seit längerer Zeit zum beruflichen Alltag (z.B. Luftfahrt- 
Hier steht eine Anzeige.

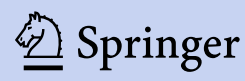




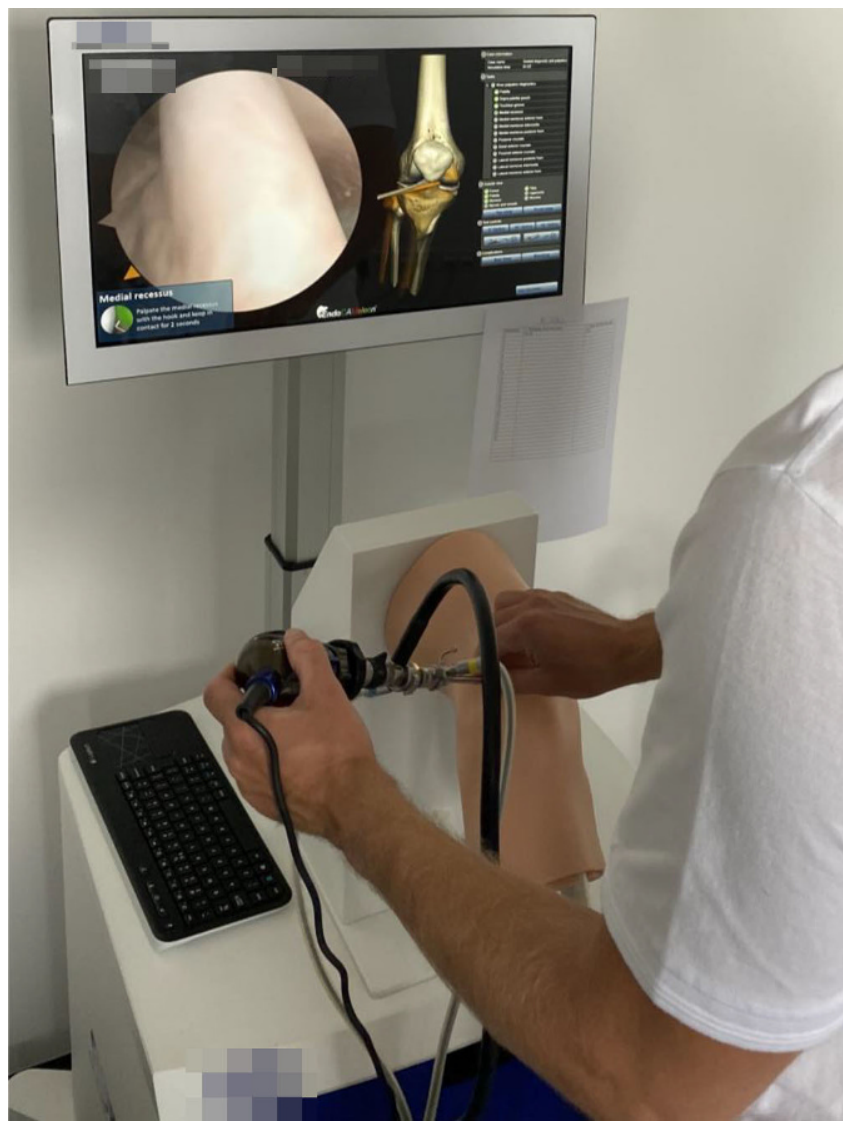

Abb. $1 \Delta$ Beispiel für einen Desktop-Gelenk-Simulator (ArthroS, Virtamed, Zürich vertrieben auch als Arthrotrainer, Karl Storz, Tuttlingen). Der Arthrotrainer ist aufgebaut wie ein arthroskopischer Arbeitsplatz mit Bildschirm, Computereinheit, Kamera und einem anatomischen Gelenkmodell (Knie, Schulter, Hüfte und Sprunggelenk) mit vorgelegten Arbeitsportalen

branche). Hier wird das Simulationstraining nicht nur zu Ausbildungszwecken verwendet, es dient auch der regelmäBigen Kontrolle der berufsspezifischen Fähigkeiten nach abgeschlossener Ausbildung.

Dieser Übersichtsartikel soll einen Überblick über den bisherigen Stand zum Einsatz virtueller Simulatoren in der chirurgischen Ausbildung in Orthopädie und Unfallchirurgie geben und eine Antwort auf die Frage nach deren wissenschaftlichen Nutzen finden.

\section{Desktop-Gelenkmodell-VR- Simulatoren}

Die längsten Erfahrungen mit virtuellen Laparoskopiesimulatoren bestehen in der Bauchchirurgie [1]. Am Beispiel der Cholezystektomie konnte bereits in den 1990er-Jahren gezeigt werden, dass die Lernkurve durch das Training am virtuel-

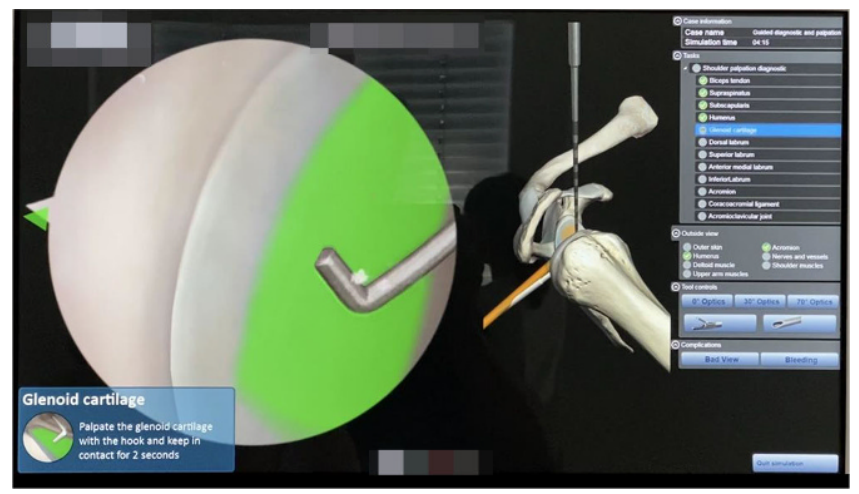

Abb. 2 ム Übung zum diagnostischen Rundgang im Gelenk am Beispiel des Schultermoduls des Arthrotrainer (Karl Storz, Tuttlingen). Definierte anatomische Strukturen können mit der virtuellen Optik eingestellt und identifiziert werden. Am linken unteren Bildrand wird die darzustellende Struktur genannt. Die Erfolgskontrolle wird über einen Farbwechsel der Struktur angezeigt (gelb nach grün)

Diese virtuellen Arthroskopiesimulatoren zeichnen sich durch anatomisch realistische Kniemodelle aus, welche auf die zweidimensionale Bildschirmoberfläche übertragen werden. Die arthroskopischen Aufgaben werden mit dem Replikationsarthroskop und den Replikationsinstrumenten in einem Gelenksimulator durchgeführt, der die taktile Rückkopplung geben kann. Dieser ist in der Regel manipulierbar, sodass auch das Weiten des Gelenkspaltes am Knie, z. B. durch Varus- und Valgusstress, simuliert werden kann. Auch Bewegungen lassen die Gelenksimulatoren zu. Die meisten Systeme können dem Anwender ferner Rückkopplung zu bestimmten Erfolgskriterien geben (Operationszeit, Kamera-/Instrumentenpfadlänge, $\mathrm{Be}$ rührungen der Knorpeloberfläche).

Im deutschsprachigen Raum finden das ArthroS (Virtamed, Zürich) oder der Arthrotrainer (Karl Storz, Tuttlingen) die breiteste Anwendung (- Abb. 1, 2, 3, 4, 5 und 6). Aus diesem Grund soll der Arthrotrainer hier genauer vorgestellt werden.

\section{Immersive virtuelle Realität}

Eine neue Art der Simulation setzt auf immersive (eintauchende) virtuelle Realität (z. B. Precision OS). Immersion beschreibt den durch eine Umgebung der VR hervorgerufenen Effekt, der das Bewusstsein des Nutzers, illusorischen Stimuli ausgesetzt zu sein, soweit in den 
Hintergrund treten lässt, dass die virtuelle Umgebung als real empfunden wird. Immersive VR ist aus dem Bereich des Gaming bekannt in dem sich Spieler vertreten durch Avatare in virtuelle Welten begeben.

Der übende Chirurg trägt eine VRBrille (z.B. Oculus Quest) mit Headset, die Bilder des Operationssaals und des Patienten projiziert und so ein realistisches Erlebnis schafft. Sensoren erfassen die Bewegungen der Hände und Finger, welche die virtuellen Instrumente während der Operation manipulieren. Um das Eintauchen in die virtuelle Realität zu fördern, ermöglichen Controller mit Tastsinn-Feedback (Haptik) die Interaktion mit Objekten in der virtuellen Welt.

\section{》) Der Teilnehmer tritt der Applikation meist als Avatar bei}

Im Vergleich zu chirurgischen „Desktop“-VR-Simulationen zeichnet sich die immersive virtuelle Realität durch naturgetreue Nachbildungen nicht nur der Anatomie, sondern auch der OPUmgebung aus. Der Teilnehmer tritt der Applikation dabei meist als Avatar bei (• Abb. 7). Die Systeme können außerdem die Anwesenheit und $\mathrm{Zu}$ sammenarbeit von mehreren Avataren gleichzeitig im virtuellen OP ermöglichen. Das bedeutet, dass der Chirurg mit anderen Chirurgen, Studenten und Instrumenteuren etc. im selben Operationssaal zusammenarbeiten kann, selbst dann, wenn sich die Personen physisch an anderen Orten befinden. Daher ist dieses System auch $\mathrm{zu}$ ortsübergreifenden Fortbildungszwecken einsetzbar (Webinare, Online-OP-Kurse etc.). Das sind Möglichkeiten, die insbesondere jetzt zur Zeit der SARS-CoV-2-Pandemie in den Vordergrund rücken [6, 25].

Aufgrund der virtuellen Nachbildung des Operationssaals und der Umgebung eignen sich immersive Systeme besonders zur Simulation offener Operationen (z.B. Hüftendoprothetik, Schulterendoprothetik, Femurmarknagel etc).

Arthroskopie 2021 · 34:94-102 https://doi.org/10.1007/s00142-020-00429-4

(c) Springer Medizin Verlag GmbH, ein Teil von Springer Nature 2021

W. Petersen - T. Hees · J. Ohde

\section{Aktueller Stand virtueller Simulatoren für die chirurgische Ausbildung in Orthopädie und Unfallchirurgie}

\section{Zusammenfassung}

Die praktische chirurgische Ausbildung wird in der Orthopädie und Unfallchirurgie durch den steigenden Kostendruck in den Kliniken sowie die Verlagerung vieler Operationen in den ambulanten Bereich immer schwieriger. Das betrifft besonders die Arthroskopie, die zudem hohe Anforderungen an die Fähigkeiten des Operateurs stellt. In den letzten 20 Jahren wurden daher zahlreiche Arthroskopiesimulatoren entwickelt. Dabei handelt es sich um virtuelle Desktopsysteme, die mit einem Gelenksimulator gekoppelt sind und eine taktile Rückkopplung erlauben. Auf diese Weise können arthroskopische Basisfähigkeiten (z. B. Triangulation) oder auch arthroskopische Operationen (z. B. partielle Meniskektomie) trainiert werden. Die Systeme ermöglichen eine Erfolgskontrolle durch die Erfassung verschiedener Parameter (Operationszeit, Kamera-/Instrumentenpfadlänge, Knorpelberührungen). Es existieren bereits Simulatoren für alle großen Gelenke. Mittlerweile liegen Daten zur Augenschein- validität, Inhaltsvalidität, Konstruktvalidität und zur Transfervalidität dieser Systeme vor. Die erlernten Fähigkeiten lassen sich daher auf den Erfolg im Operationssaal übertragen. Ungelöst ist bisher die Kosteneffizienz dieser Systeme. Von den Desktop-GelenkmodellSystemen müssen immersive VirtualReality(VR)-Systeme unter Verwendung von 3-D-Brillen und taktilen Handsensoren abgegrenzt werden. Der Teilnehmende tritt der Applikation dabei meist als Avatar bei. Daher eignen sich immersive Systeme besonders zur Simulation offener Operationen (z. B. Hüftendoprothetik). Erste Studien haben auch für den Einsatz der immersiven virtuellen Realität Evidenz für Inhalts-, Konstrukt- und Transfervalidität beim Training verschiedener offener Operationen gezeigt. Hier sind in Zukunft weitere Entwicklungen zu erwarten.

Schlüsselwörter

Virtuelle Realität · Simulation · Kniegelenk · Hüfte · Schulter

\section{Current status of virtual simulators for surgical training in orthopedics and trauma surgery}

\section{Abstract}

Practical surgical training in orthopedics and trauma surgery is becoming more and more difficult due to increasing economic pressure. In addition, more and more interventions are carried out on an outpatient basis. This particularly applies to arthroscopy, which also places high demands on the skills of the surgeon. Therefore, numerous virtual arthroscopy simulators have been developed over the past 20 years. These are virtual desktop systems that are coupled with a joint simulator, which allow tactile feedback. In this way basic arthroscopic skills (e.g. triangulation) or arthroscopic operations (e.g. partial meniscectomy) can be trained. The systems enable success control by recording various parameters (surgical time, camera and instrument path length, cartilage contact). Specific simulators already exist for all major joints. Scientific data are now available on face validity, content validity, construct validity and transfer validity of these systems. The skills learned can therefore be transferred to success in the operating room. The cost efficiency of these systems has so far not been resolved. Immersive virtual reality (VR) systems using 3D goggles and tactile hand sensors must be distinguished from desktop joint model systems. The participants usually join the application as an avatar. Immersive systems are therefore particularly suitable for simulating open surgical procedures (e.g. hip arthroplasty). Initial studies have also shown evidence for the use of immersive virtual reality for content, construct and transfer validity when training various open operations. Further developments are to be expected here in the future.

Keywords Virtual reality · Simulation · Knee joint · Hip . Shoulder 


\begin{tabular}{|c|c|c|c|c|}
\hline & System/Firma & Typ & Applikationen & Feedback \\
\hline 1 & Sheffield Knee Arthroscopy Training System (SKATS) & Desktop VR puls Gelenksimulator & Knie, Schulter & Visuell, taktil \\
\hline 2 & ARTHRO Mentor/Symbionix, Cleveland, $\mathrm{OH}$ & Desktop VR puls Gelenksimulator & Knie, Schulter, Hüfte & Visuell, taktil \\
\hline 3 & $\begin{array}{l}\text { ArthroS/Virtamed, Zurich, Switzerland oder Arthrotrai- } \\
\text { ner, Karl Storz, Tuttlingen }\end{array}$ & Desktop VR puls Gelenksimulator & $\begin{array}{l}\text { Knie, Schulter, Hüfte oberes } \\
\text { Sprunggelenk }\end{array}$ & Visuell, taktil \\
\hline 4 & ArthroSim/Touch of Life Technologies, Aurora, CO & Desktop VR puls Gelenksimulator & Knie, Schulter & Visuell, taktil \\
\hline 5 & InsightARTHRO VR ${ }^{\circledR}$ (3D Systems, Valencia, USA) & Desktop VR puls Gelenksimulator & Knie, Schulter & Visuell, taktil \\
\hline 6 & Precision OS & $\begin{array}{l}\text { Immersive VR mit VR-Brille und takti- } \\
\text { lem "Controller" }\end{array}$ & Knie, Schulter & Visuell, taktil \\
\hline 7 & THA VR Simulation, Pixelmolkerei, Chur, Switzerland & $\begin{array}{l}\text { Immersive VR mit VR-Brille und takti- } \\
\text { lem „Controller" }\end{array}$ & Hüfte & Visuell, taktil \\
\hline
\end{tabular}

\section{Gibt es wissenschaftliche Evidenz für virtuelle Operationssimulatoren?}

In den letzten Jahren sind zahlreiche Studien erschienen, die sich mit der Frage nach der wissenschaftlichen Evidenz virtueller Simulationsgeräte für chirurgische Operationen befasst haben.

Im orthopädischen Bereich beschäftigen sich die meisten Arbeiten mit virtuellen Desktop-Arthroskopiesimulatoren. Es liegen erst wenige Arbeiten zu immersiven virtuellen Simulatoren unter Verwendung von VR-Brillen vor. Beide Systeme sollen daher im Hinblick auf ihre wissenschaftliche Evidenz getrennt betrachtet werden.

\section{Evidenz für virtuelle Desktop- Gelenkmodell-Simulatoren}

Für die wissenschaftliche Evidenz von Desktop-Simulatoren in der Orthopädie liegen überwiegend Studien zum Einsatz in der arthroskopischen Ausbildung vor. Diese Studien lassen sich in verschiedene Validierungsebenen einteilen ([26, 27, 29]; - Tab. 2).

Die Augenscheinvalidität („face validity“) überprüft die Realitätsnähe der arthroskopischen Darstellung im Simulator $[27,29]$. Sie wird erfasst, indem die Probanden die Realitätstreue anatomischer Strukturen, Farben, Größe, Beschaffenheit der Instrumente etc. beispielsweise auf einer Skala (1-10) bewerten [23]. Verschiedene aktuelle Studien und systematische Reviews konnten zeigen, dass die gängigen Arthroskopiesimulatoren eine hohe Realitätsnähe (Augenscheinvalidität) haben $[5,13,14,23,24,26]$. Eine
Studie konnte jedoch Unterschiede unter drei verschiedenen virtuellen Arthroskopiesimulatoren zeigen. In dieser Studie schnitt das ArthroS-System (Virtamed) hinsichtlich der externen "face validity“ am besten ab [23].

\section{》) Inhaltsvalidität bezeichnet die Messung verschiedener arthroskopischer Fähigkeiten}

Inhaltsvalidität („content validity“) bezeichnet die Messung verschiedener arthroskopischer Fähigkeiten [26, 27, 29]. Am besten eignen sich dazu objektive Messparameter (z. B. Zeit, Kamerapfadlänge, Horizonteinstellung, Knorpelberührungen/Schäden etc.), die vom virtuellen System erfasst und quantifiziert werden können. So können die arthroskopischen Fähigkeiten erfasst und der Lernfortschritt der Übenden oder Probanden verfolgt werden (Lernkurve). Der klinisch am häufigsten verwendete Messparameter, um eine Lernkurve $\mathrm{zu}$ beschreiben, ist die Zeit. Auch die „content validity“ moderner und gebräuchlicher virtueller Arthroskopiesimulatoren ist wissenschaftlich belegt. So konnten verschiedene Studien zeigen, dass sich die für die jeweilig abgefragte arthroskopische Aufgabe benötigte Zeit insbesondere bei Anfängern mit der Anzahl der Wiederholungen verkürzt [27]. Bei Fortgeschrittenen war dieser Effekt nicht in allen Studien signifikant [26, 29].

Die Konstruktvalidität gibt ein Maß an für die Eignung des Systems, anhand objektiver Messparameter unterschiedliche „skills level“ von Arthroskopeuren oder Probanden zu unterscheiden. Ver- schiedene Studien konnten bestätigen, dass verschiedene Messparameter geeignet sind, das Leistungsniveau von Anfängern, Fortgeschrittenen und Experten zu unterscheiden [7, 13, 19, 26, 27].

Die größte klinische Relevanz hat die Transfer- oder Übertragungsvalidität. Die Transfervalidität erlaubt die Aussage darüber, ob die am Simulator erlernten arthroskopischen Fähigkeiten auch die Leistung im Operationssaal verbessern. Außerdem ist von Relevanz, inwieweit die erlernten Fähigkeiten auch über die Zeit beibehalten werden. Ein aktuelles systematisches Review konnte zwei randomisierte kontrollierte Studien identifizieren, welche die Transfervalidität eines virtuellen arthroskopischen Simulatortrainings untersucht haben [9]. Die erste Studie zeigte eine verbesserte operative Leistung bei grundlegenden arthroskopischen Aufgaben am Knie im Operationssaal nach einer festgelegten Trainingsperiode am virtuellen Simulator. Der Unterschied im Leistungszuwachs zur nichttrainierten Kontrollgruppe war signifikant [9]. Auch die zweite Studie konnte zeigen, dass sich die Leistung bei arthroskopischen Aufgaben im Operationssaal durch ein arthroskopisches Simulatortraining verbessern ließ [9]. Zudem zeigte sich in dieser Studie keine weitere Verschlechterung des Kompetenzniveaus in einem Zeitraum von 6 Monaten nach dem Simulatortraining [9]. Darüber hinaus konnten beide Studien zeigen, dass die technischen Möglichkeiten der Simulatoren zur Bewegungsanalyse für die Bewertung technischer Fähigkeiten relevant sind. Die Zeit bis zur Erledigung der Aufgabe, der Weg der Instrumente und das Auftre- 


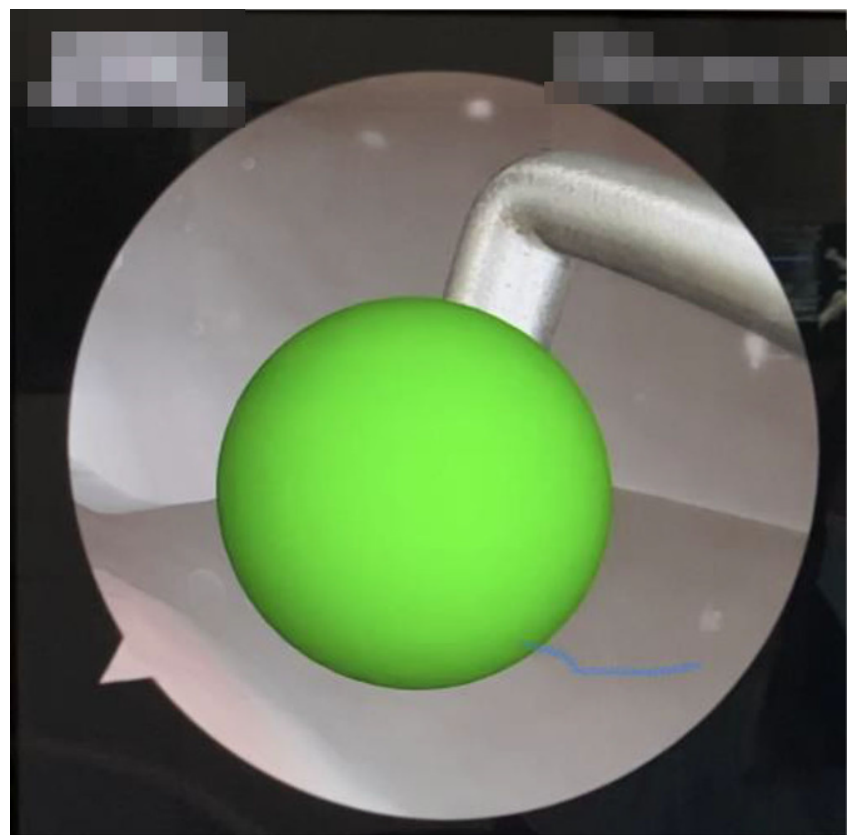

Abb. 3 ॥ Übung zur Triangulation am Arthrotrainer mit Kniemodell. Eine Triangulationsübung ist das Berühren virtueller Kugeln mit dem Tasthaken

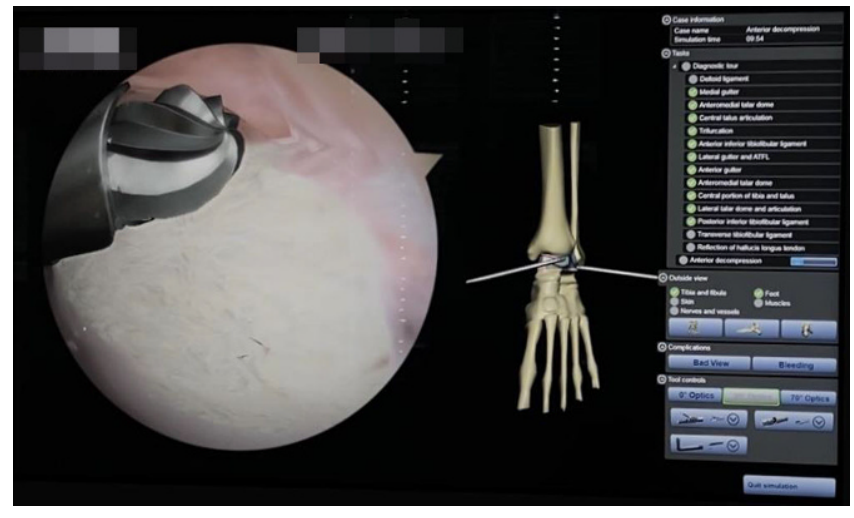

Abb. $5<$ Je nach Gelenk sind auch verschiedene realistische und typische arthroskopische Prozeduren durchführbar (hier dargestellte die tibiale Spornabtragung am oberen Sprunggelenk)

ten von Instrumentenkollisionen waren mit der höchsten Validität und Zuverlässigkeit verbunden [9]. Die Ergebnisse der beiden Studien zeigen, dass das Training mit dem Kniearthroskopiesimulator zu einer Leistungsverbesserung führen kann.

Zur prognostischen Validität des virtuellen Simulatortrainings im Hinblick auf das postoperative Ergebnis liegen bisher keine Studien vor.

\section{Evidenz für immersive virtuelle Operationssimulatoren}

Für den Einsatz der immersiven virtuellen Realität in der orthopädischen chirurgischen Ausbildung liegen deutlich we- niger Studien vor als zu den Desktopsimulatoren [20-22].

Logishetti et al. haben ein immersives VR-System zur Hüftendoprothetik (THA VR Simulation, Pixelmolkerei, Chur, Switzerland) in einer kontrollierten randomisierten Studie mit 24 orthopädischen Assistenzärzten ohne vorherige Erfahrung in der Hüftendoprothetik untersucht [21]. Dabei wurde ein anteriorer Zugang durchgeführt. Von den 24 Assistenzärzten absolvierten 12 ein 6-wöchiges VR-Schulungsprogramm in einem Simulationslabor, während sich die anderen 12 Assistenzärzte konventionell auf die Operation vorbereiteten. Alle Probanden führten anschließend eine Hüftprothesenimplantation an einem
Körperspender durch, die unabhängig voneinander von zwei Hüftchirurgen beurteilt wurde (THA-spezifische verfahrensbasierte Bewertung). Die Auswertung der Studie zeigte, dass VR-geschulte Chirurgen in der Bewertung ein höheres chirurgisches Niveau erreichten als die Kontrollprobanden (Stufe 3a vs. Stufe 2a). Außerdem waren VR-geschulte Chirurgen um $12^{\circ}$ genauer in der Komponentenorientierung und waren $18 \%$ schneller ( $42 \mathrm{~min}$ vs. $51 \mathrm{~min}$ ). Damit konnte diese Studie Inhalts- und Transfervalidität für immersive VRSimulation in der Hüftendoprothetik zeigen [21].

Die gleiche Arbeitsgruppe hat eine zweite randomisierte Studie mit 32 Assistenzärzten durchgeführt [20]. In dieser Studie konnte gezeigt werden, dass die VR-geschulten Probanden die chirurgischen Fähigkeiten durch wiederholtes Üben schrittweise verbesserten (Lernkurve, Inhaltsvalidität). Nach durchschnittlich 4 Sitzungen war ein Plateau erreicht. VR-geschulte Probanden erreichten in 9 von 10 Kriterien den höchsten Level in der Bewertung der Operationen (mit Ausnahme des femoralen Osteotomiewinkels). Fehler wurden um $79 \%$, unterstützende Eingabeaufforderungen um $70 \%$ und die Operationsdauer um $28 \%$ reduziert. Die femorale Osteotomie wurde genauer und die Ausrichtung des Azetabulumimplantats präziser durchgeführt. Damit konnte diese Studie neben der Inhaltsund Transfervalidität auch Konstruktvalidität für immersive VR-Applikationen 

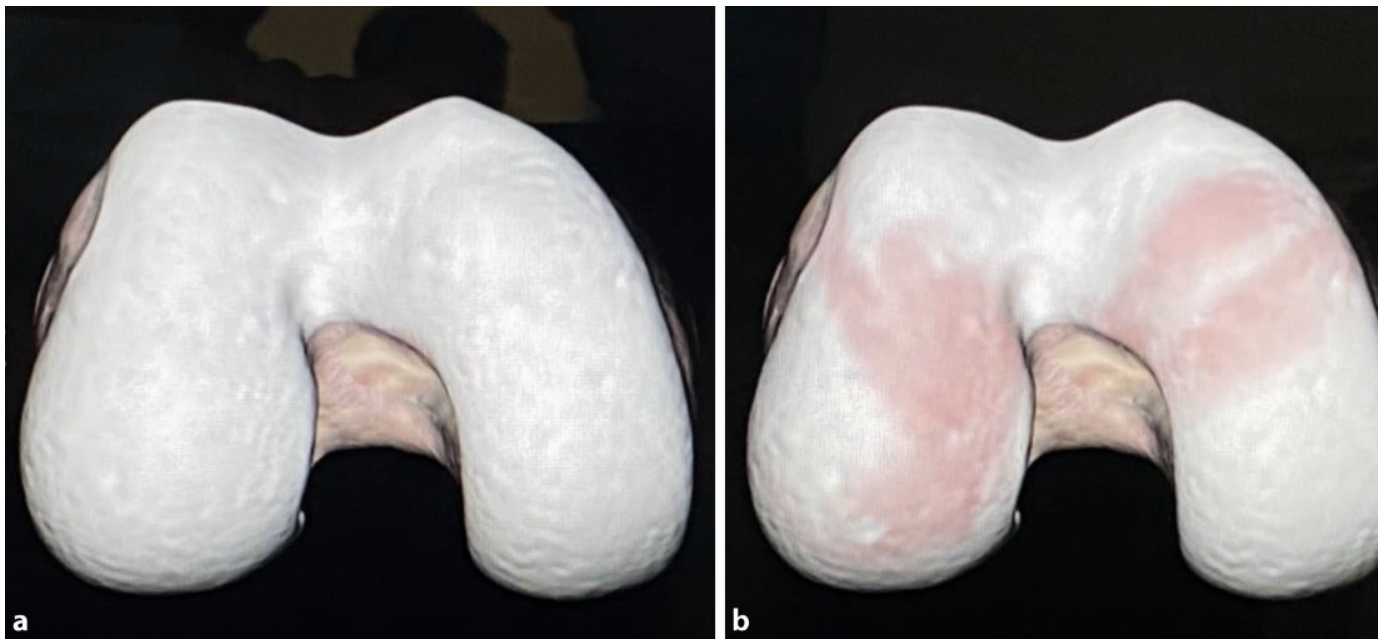

Abb. $6<$ Neben den verschiedenen Messparametern (Operationszeit, Kamera-/ Instrumentenpfadlänge) kann die mit dem Arthroskop und den Instrumenten berührte Knorpelfläche prozentual erfasst werden (a vor der Behandlung, b nach der Behandlung)

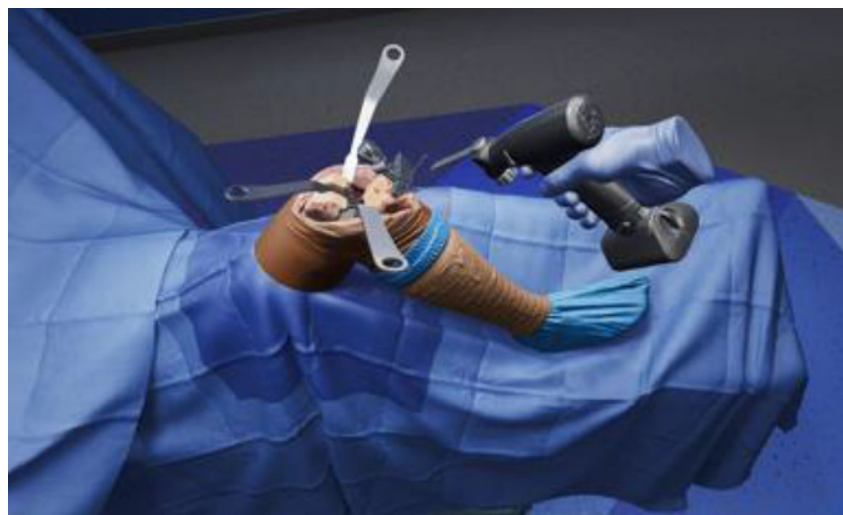

Abb. 7 ॥ Beispiel für eine Anwendung immersiver virtueller Realität (VR). Dargestellt ist eine offene Revisionsknieprothesenoperation. Im Unterschied zu den Desktop-Gelenk-Simulatoren wird auch der Operationssaal nachgebildet. Der Teilnehmer der virtuellen Übung tritt der Operation als Avatar bei. (Mit freundlicher Genehmigung PrecisionOS, Seattle/USA)

in der chirurgischen Ausbildung aufzeigen [20].

Die dritte klinische Studie (multizentrisch, randomisiert) zur immersiven VR-Simulation hat sich mit der Schulterendoprothetik beschäftigt [22]. An dieser Studie nahmen 19 Assistenzärzte und 7 in der Schulterendoprothetik erfahrene Chirurgen teil. Die für das Ergebnis der Studie relevante Aufgabe konzentrierte sich auf die Glenoidexposition. Die „VR-Performance“ wurde mit der Glenoidexposition an Leichenpräparaten verglichen. In dieser Studie zeigte immersive VR einen größeren Realismus als die Kontrolle („face validity“). Die immersive VR-Gruppe absolvierte die Lerninhalte signifikant schneller als die Kontrollgruppe $(14 \pm 7 \mathrm{~min}$ vs. $21 \pm 6 \mathrm{~min})$. Auch in der Bewertung der Instrumentenhandhabung war die immersive VR-Gruppe überlegen. Damit konnte diese Studie Augenschein-, Inhalts-, Konstrukt- und Transfervalidität für immersive VR in der orthopädisch chirurgischen Ausbildung zeigen [22].

Zusammenfassend zeigen diese drei kontrollierten randomisierten Studien eine hohe Evidenz für den Einsatz immersiver VR-Simulation in der chirurgischen Ausbildung beim Gelenkersatz an Schulter und Hüfte.

\section{Diskussion}

Für den Einsatz virtueller Simulatoren in der orthopädisch chirurgischen Ausbildung liegt mittlerweile ausreichend Evidenz vor. Das gilt für Desktop-GelenkSimulatoren und auch für immersive VR. Aufgrund der nachgewiesenen Transfer- validität tragen diese Systeme damit auch zu einer erhöhten Patientensicherheit bei.

In arthroskopischen Operationskursen werden virtuelle Simulatoren zwar bereits genutzt und die AGA hat die „virtuelle Arthroskopie“ bereits in ihr Aus- und Weiterbildungskonzept eingebunden (http://www.aga-online.ch/ fortbildung/aga-simulatortrainingarthro skopie-start/, www.arthroskopie-sylt. de). Trotzdem erfolgt der Einzug des Simulatortrainings in den klinischen Alltag zu zögerlich. Da in den meisten Studien zur Transfervalidität die Übungsserien über einen mehrwöchigen Zeitraum durchgeführt wurden, wäre es wünschenswert, wenn chirurgische Simulatoren auch in Ausbildungskliniken verfügbar wären. Die Inhaltsvalidität dieser Systeme legt auch eine Anwendung in der Überwachung von chirurgischen Fähigkeiten im Langzeitverlauf analog zu anderen Berufen wie den Routinechecks von Piloten in der Luftfahrt nahe.

Ein Grund für die fehlende Akzep$\operatorname{tanz}$ dieser Systeme in der Krankenhauslandschaft sind sicher die hohen Anschaffungskosten von Desktop-Gelenk-Simulatoren. Diese liegen für den Arthrotrainer (Karl Storz) derzeit bei ca. 100.000 EUR. Studien zur Kosteneffizienz von chirurgischen Trainingssimulatoren auf dem Gebiet der Orthopädie und Unfallchirurgie fehlen [7, 26, 29]. Es liegen jedoch aus anderen Fachgebieten Hinweise auf eine Kosteneffizienz dieser Systeme vor. Für die Laparoskopie wurde in einer amerikanischen Studie kalkuliert (Ausbildungszeit, Operations- 
Tab. 2 Validierungsstufen für virtuelle Desktop-Simulatoren. (Nach Angaben von Tay et al. [29] und Rashed et al. [26])

\begin{tabular}{l} 
Validitätsstufe \\
\hline Augenscheinvalidität \\
Inhaltsvalidität („,con- \\
tent validity“)
\end{tabular}

Konstruktvalidität ("construct validity")

Transfervalidität ("transfer validity“)

\section{Relevanz}

Externe und intraartikuläre Realitätsnähe des Simulators

Messung verschiedener arthroskopischer Fähigkeiten (Zeit, Kamerapfadlänge, Horizonteinstellung, Knorpelberührungen/Schäden etc.), Erfassung von Lernkurven

Eignung des Systems, anhand objektiver Messparameter unterschiedliche "skills level“" von Arthroskopeuren oder Probanden zu unterscheiden Verbesserung der Leistung im Operationssaal durch die am Simulator erlernten arthroskopischen Fähigkeiten Beibehaltung der erlernten Fähigkeiten über die Zeit zeit, weniger Kosten für Ausbilder etc.), dass ein Simulatorsystem in 6 Monaten ca. 160.000 US-Dollar einsparte und innerhalb von 131 Tagen seine Investition zurückgab [18]. Kritisch ist hier anzumerken, dass derartige Kalkulationen stark vom jeweiligen Gesundheitssystem abhängen und auch nicht immer von einer Einrichtung auf die andere übertragbar sind. Es fehlen auch Vergleiche $\mathrm{zu}$ anderen, deutlich weniger kostenintensiven Simulatoren (Zigarrenbox-Simulator; [28]. Im Hinblick auf die Evidenz der Kosteneffizienz chirurgischer Lernsimulatoren besteht auf dem Gebiet der Orthopädie und Unfallchirurgie großer wissenschaftlicher Nachholbedarf.

Im Vergleich zu den Desktop-GelenkSimulatoren auf dem Gebiet der arthroskopischen Chirurgie sind immersive Systeme zum virtuellen Training von Operationen weit weniger verbreitet. Das kann daran liegen, dass die Entwicklung dieser Systeme später begann. Hier sind wahrscheinlich noch weitere Entwicklungen mit deutlichen Fortschritten in der Realitätsnähe dieser Systeme und auch weitere Applikationen zu erwarten. Derzeit wird die immersive VR hauptsächlich für das Training offener Operationen verwendet. Anwendungen in der Arthroskopie erscheinen trotzdem möglich. Ein wesentliches Argument für immersive VR wird ökonomischer Art sein. Immersive VRSysteme sind deutlich kostengünstiger als Desktop-Gelenk-Simulatoren.

Im Hinblick auf die Diskussion um die Kosteneffizienz von Ausbildung in chirurgischen Fächern wäre zu fordern, dass von gesundheitspolitischer Seite stärkere finanzielle Anreize geschaffen werden, um die chirurgische Ausbildung zu för- dern und die Patientensicherheit zu erhöhen.

\section{Fazit für die Praxis}

\section{- Virtuelle Simulatoren erweitern die Möglichkeiten in der Chirurgie deut- lich und arthroskopische VR(Virtual- Reality)-Gelenksimulatoren haben bereits Einzug in die arthroskopische Ausbildung genommen. \\ - Wissenschaftliche Daten liegen zu fast allen großen Gelenken vor. \\ - Ungelöst ist bisher die Kosteneffizi- enz dieser Systeme. \\ - Auch erste immersive VR-Systeme ha- ben ihre Evidenz für die Anwendung bei offenen Operationen gezeigt. \\ - Hier sind in Zukunft weitere Entwick- lungen zu erwarten.}

\section{Korrespondenzadresse}

\section{Prof. Dr. med. Wolf Petersen}

Sportklinik Berlin und Klinik für Orthopädie und Unfallchirurgie, am Martin Luther Krankenhaus, Berlin-Grunewald

Caspar-Theyß-Straße 27-31, 14193 Berlin, Deutschland

wolf.petersen@jsd.de

\section{Einhaltung ethischer Richtlinien}

Interessenkonflikt. W. Petersen: Beratertätigkeit für die Firmen Karl Storz, Otto Bock und OPED. T. Hees und J. Ohde geben an, dass kein Interessenkonflikt besteht.

Für diesen Beitrag wurden von den Autoren keine Studien an Menschen oder Tieren durchgeführt. Für die aufgeführten Studien gelten die jeweils dort angegebenen ethischen Richtlinien.

\section{Literatur}

1. Ahlberg G, Enochsson L, Gallagher AG et al (2007) Proficiency-based virtual reality training significantly reduces the error rate for residents during their first 10 laparoscopic cholecystectomies. Am J Surg 193:797-804. https://doi.org/10.1016/j. amjsurg.2006.06.050

2. Aïm F, Lonjon G, Hannouche D, Nizard R (2016) Effectiveness of virtual reality training in orthopaedic surgery. Arthroscopy 32:224-232. https://doi. org/10.1016/j.arthro.2015.07.023

3. Alvand A, Auplish S, Gill H, Rees J (2011) Innate arthroscopic skills in medical students and variation in learning curves. J Bone Joint Surg Am 93:e115(1). https://doi.org/10.2106/JBJS.K.00199

4. Alvand A, Auplish S, Khan T et al (2011) Identifying orthopaedic surgeons of the future-The inability of some medical students to achieve competence in basic arthroscopic tasks despite training: A randomised study. J Bone Joint Surg $\mathrm{Br}$ 93 B:1586-1591. https://doi.org/10.1302/0301620X.93B12.27946

5. Angelo RL, Ryu RKN, Pedowitz RA et al (2015) A proficiency-based progression training curriculum coupled with a model simulator results in the acquisition of a superior arthroscopic Bankart skill set. Arthroscopy 31:1854-1871. https://doi.org/ 10.1016/j.arthro.2015.07.001

6. Backhaus L, Bierke S, Karpinski K et al (2020) Übersichten SARS-CoV-2-Pandemie und ihre Auswirkungen auf Orthopädie und Unfallchirurgie: "Booster" für die Telemedizin Einleitung. Knie J. https://doi.org/10.1007/s43205-020-00062-z

7. Bartlett JD, Lawrence JE, Stewart ME et al (2018) Does virtual reality simulation have a role in training trauma and orthopaedic surgeons? Bone Joint J 100B:559-565. https://doi.org/10.1302/ 0301-620X.100B5.BJJ-2017-1439

8. Bert JM (2016) First, do no harm: protect the articular cartilage when performing arthroscopic knee surgery! Arthroscopy 32:2169-2174. https:// doi.org/10.1016/j.arthro.2016.07.023

9. Boutefnouchet T, Laios T (2016) Transfer of arthroscopic skills from computer simulation training to the operating theatre: a review of evidence from two randomised controlled studies. SICOT J 2:4. https://doi.org/10.1051/sicotj/ 2015039

10. Compton J, Slattery M, Coleman M, Westermann R (2020) latrogenic articular cartilage injury in arthroscopic hip and kneevideos and the potenzial for cartilage cell death when simulated in a bovine model. Arthroscopy 36:2114-2121. https://doi. org/10.1016/j.arthro.2020.02.017

11. Cunningham $G$, Benchouk $S$, Kherad $O$, Lädermann A (2016) Comparison of arthroscopic and open Latarjet with a learning curve analysis. Knee Surg Sports Traumatol Arthrosc 24:540-545. https://doi.org/10.1007/s00167-015-3910-3

12. Dietrich F, Ries C, Eiermann C et al (2014) Complications in hip arthroscopy: necessity of supervision during the learning curve. Knee Surg Sports Traumatol Arthrosc 22:953-958. https:// doi.org/10.1007/s00167-014-2893-9

13. Frank RM, Wang KC, Davey A et al (2018) Utility of modern arthroscopic simulator training models: a meta-analysis and updated systematic review. Arthroscopy 34:1650-1677. https://doi.org/10. 1016/j.arthro.2017.10.048

14. Fucentese SF, Rahm S, Wieser K et al (2015) Evaluation of a virtual-reality-based simulator using passive hapticfeedbackfor knee arthroscopy. Knee Surg Sports Traumatol Arthrosc 23:1077-1085. https://doi.org/10.1007/s00167-014-2888-6 
15. Hoppe DJ, De Sa D, Simunovic N et al (2014) The learning curve for hip arthroscopy: a systematic review. Arthroscopy 30:389-397. https://doi.org/ 10.1016/j.arthro.2013.11.012

16. Kim SJ, Moon HK, Chun YM, Chang JH (2011) Arthroscopic treatment for limitation of motion of the elbow: the learning curve. Knee Surg Sports Traumatol Arthrosc 19:1013-1018. https://doi. org/10.1007/s00167-010-1334-7

17. Koehler RJ, Amsdell S, Arendt EA et al (2013) The Arthroscopic Surgical Skill Evaluation Tool (ASSET). Am J Sports Med 41:1229-1237. https://doi.org/ $10.1177 / 0363546513483535$

18. Kunkler K (2006) The role of medical simulation: an overview. Int J Med Robot 2:203-210. https://doi. org/10.1002/rcs.101

19. Laprell H, Stein V, Petersen W (2002) Arthroscopic all-inside meniscus repair using a new refixation device: a prospective study. Arthroscopy 18:387-393. https://doi.org/10.1053/jars.2002. 30639

20. LogishettyK, Gofton WT, Rudran B et al (2020) Fully immersive virtual reality for total hip arthroplasty: objective measurement of skills and transfer of visuospatial performance after a competencybased simulation curriculum. JBone Joint Surg Am. https://doi.org/10.2106/JBJS.19.00629

21. Logishetty K, Gofton WT, Rudran B et al (2020) A multicenter randomized controlled trial evaluating the effectiveness of cognitive training for anterior approach total hip arthroplasty. J Bone Joint Surg Am 102:e7. https://doi.org/10.2106/JBJS.19. 00121

22. Lohre R, Bois AJ, Athwal GS, Goel DP (2020) Improved complex skill acquisition by immersive virtual reality training: a randomized controlled trial. J Bone Joint Surg Am. https://doi.org/10. 2106/JBJS.19.00982

23. Martin KD, Akoh CC, Amendola A, Phisitkul P (2016) Comparison of three virtual reality arthroscopic simulators as part of an orthopedic residency educational curriculum. lowa Orthop J 36:20-25

24. Morgan M, Aydin A, Salih A et al (2017) Current status of simulation-based training tools in orthopedic surgery: a systematic review. J Surg Educ 74:698-716. https://doi.org/10.1016/j.jsurg. 2017.01.005

25. Petersen W, Bierke S, Karpinski K, Häner M (2020) Coronavirus-Pandemie und ihre Auswirkungen auf Orthopädie und Unfallchirurgie: Operationen, Risiken und Prävention? Knie J. https://doi.org/10. 1007/s43205-020-00052-1

26. Rashed S, Ahrens PM, Maruthainar N et al (2018) The role of arthroscopic simulation in teaching surgical skills: a systematic review of the literature. JBJS Rev 6:e8. https://doi.org/10.2106/JBJS.RVW. 17.00201

27. Reppenhagen S, Weißenberger M, Barthel T et al (2019) Virtual arthroscopy: Gaming or training concept of the future. Unfallchirurg 122:431-438. https://doi.org/10.1007/s00113-019-0653-5

28. Sandberg RP, Sherman NC, Latt LD, Hardy JC (2017) Cigar box arthroscopy: a randomized controlled trial validates nonanatomic simulation training of novice arthroscopy skills. Arthroscopy 33:2015-2023.e3. https://doi.org/10.1016/j. arthro.2017.04.022

29. Tay C, Khajuria A, Gupte C (2014) Simulation training: a systematic review of simulation in arthroscopy and proposal of a new competencybased training framework. Int J Surg 12:626-633. https://doi.org/10.1016/j.ijsu.2014.04.005

30. Vega J, Golanó P, Peña F (2016) latrogenic articular cartilage injuries during ankle arthroscopy. Knee Surg Sports Traumatol Arthrosc 24:1304-1310. https://doi.org/10.1007/s00167-014-3237-5

\section{Aktuelle Empfehlungen aus dem Springer-Verlag}

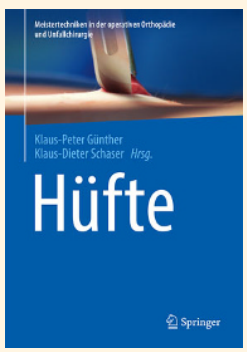

\section{Hüfte}

K.-P. Günther, K.-D. Schaser (Hrsg.)

1. Aufl. 2021, VIII, 378 S., 268 Abb., Hardvoer 149,99€

ISBN 978-3-662-62084-7

Wesentliche Operationsverfahren werden step-by-step dargestellt und mit zahlreichen Fotos und brillanten Grafiken visualisiert.

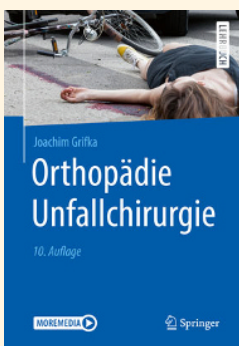

\section{Orthopädie Unfallchirurgie}

\section{J. Grifka}

10. Aufl. 2020, XII, 625 S., 533 Abb., Softcoer 39,99€

ISBN 978-3-662-60519-6

Highlicht der komplett aktualisierten und überarbeiteten Neuauflage sind zahlreiche Videos, die klassische Untersuchungsmethoden anschaulich verdeutlichen.

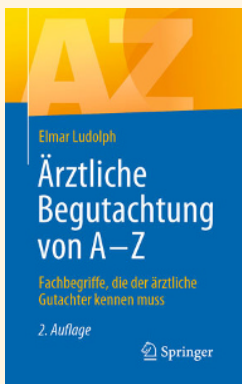

\section{Ärztliche Begutachtung von A - Z}

E. Ludolph

2. Aufl. 2020, XXXVIII, 391 S., Softcover $24,99 €$

ISBN 978-3-662-61739-7

Die wichtigsten rechtlichen Begriffe, die der ärztliche Gutachter kennen sollte, übersichtlich, kompakt und präzise erläutert.

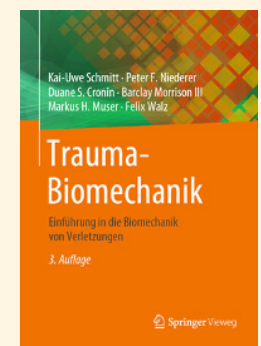

\section{Trauma-Biomechanik}

K.-U. Schmitt, P.F. Niederer, D.S. Cronin, B. Morrison, M.H. Muser, F. Walz 3. Aufl. 2020, XVI, 327 S., 138 Abb., Hardcover 79,99€ ISBN 978-3-662-60935-4

Die aktualisierte Auflage behandelt die Auswirkungen mechanischen Belastungen auf den Körper und zeigt Ansätze zur Prävention von Verletzungen auf.

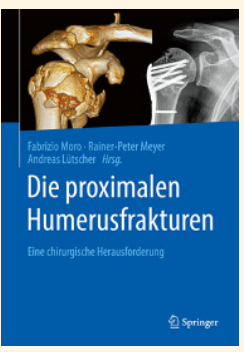

\section{Die proximalen Humerusfrakturen}

F. Moro, R.-P. Meyer, A. Lütscher (Hrsg.)

1. Aufl. 2020, XVI, 414 S., 362 Abb., Hardcover $64,99 €$ ISBN 978-3-662-60852-4

55 Fälle werden mit Blick auf Indikations-, Beurteilungs- und Behandlungsfehler analysiert - ein Plädoyer für die Weitergabe von Fällen, die das eigene handwerkliche Können übersteigen. 